\section{Mid-gut bleeding due to a chronic splanchnic vascular stricture with secondary portal hyper- tension after gastric bypass}

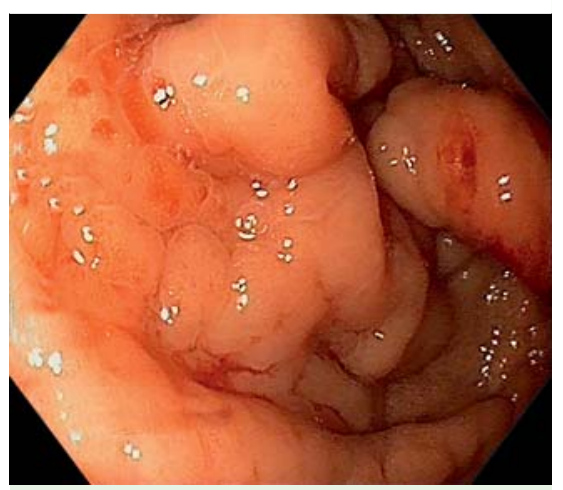

Fig. 1 Esophagogastroduodenoscopy shows severe portal hypertensive gastropathy of the fundal pouch, signs of fresh bleeding, and normal jejunal mucosa in a patient with a past history of laparoscopic Roux-en-Y gastric bypass.

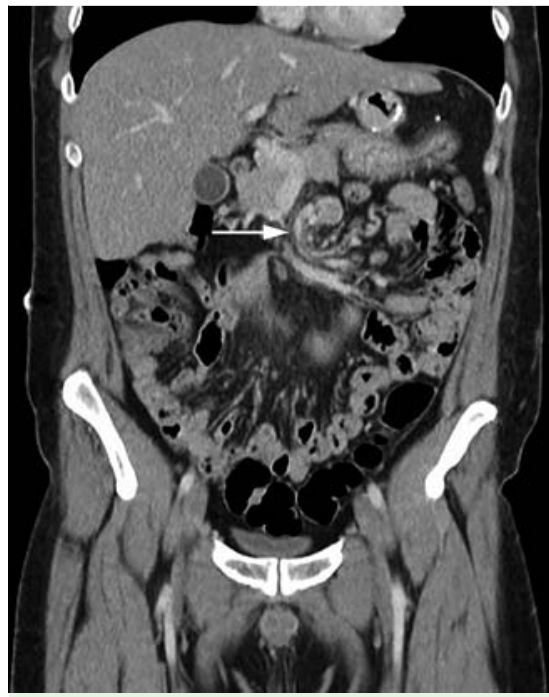

Fig. 2 Computed tomographic angiography shows a proximal mesenteric torsion, occlusion of the superior mesenteric vein (arrow) with signs of portal hypertension, and stenosis of the superior mesenteric artery.

A 41-year-old man presented with a 24hour history of melena and exacerbated epigastric pain 6 years after undergoing a laparoscopic Roux-en-Y gastric bypass for morbid obesity. He had been experiencing recurrent abdominal pain and episodes of melena in the preceding months. His past medical history also included protein $C$ deficiency and a pulmonary embolism treated with anticoagulant therapy, which had been stopped 6 months earlier following bleeding from an anastomotic ulcer. Physical examination revealed low blood pressure, tachycardia, and epigastric pain, without rebound tenderness. The patient had lost $42 \mathrm{~kg}$ since the laparoscopic Roux-en-Y gastric bypass. Laboratory data disclosed an acute fall in the hemoglobin level $(7.4 \mathrm{~g} / \mathrm{dL})$ and increased blood urea nitrogen $(50 \mathrm{mg} / \mathrm{dL})$. Esophagogastroduodenoscopy showed severe portal hypertensive gastropathy of the fundal pouch, signs of fresh bleeding, and normal jejunal mucosa ( $\bullet$ Fig.1). Emergency computed tomographic angiography showed a proximal mesenteric torsion and occlusion of the superior mesenteric vein with signs of portal hypertension ( Fig. 2).

The patient underwent a laparotomy, which revealed an internal hernia in the Petersen space. The internal hernia was reduced without bowel resection. Two weeks after closure of the Petersen space, abdominal computed tomography disclosed persistence of the superior mesenteric vein occlusion ( $\bullet$ Fig. 3). Repermeabilization of the superior mesenteric vein ( Fig.4) was achieved after 3 months of anticoagulant therapy, without complications or recurrence of the abdominal pain. Internal hernias are more common following laparoscopic Roux-en-Y gastric bypass than after open surgery [1]. In order to minimize this risk, several authors suggest closure of the mesenteric defects [2]. Actually, complete mesenteric closure after laparoscopic Roux-en-Y gastric bypass is still debated $[3,4]$.

Digestive bleeding related to a chronic splanchnic vascular stricture with secondary portal hypertension after laparoscopic Roux-en-Y gastric bypass has never been described. Surgeons and endoscopists should be aware of this rare complication.

Endoscopy_UCTN_Code_CCL_1AC_2AD

Competing interests: None

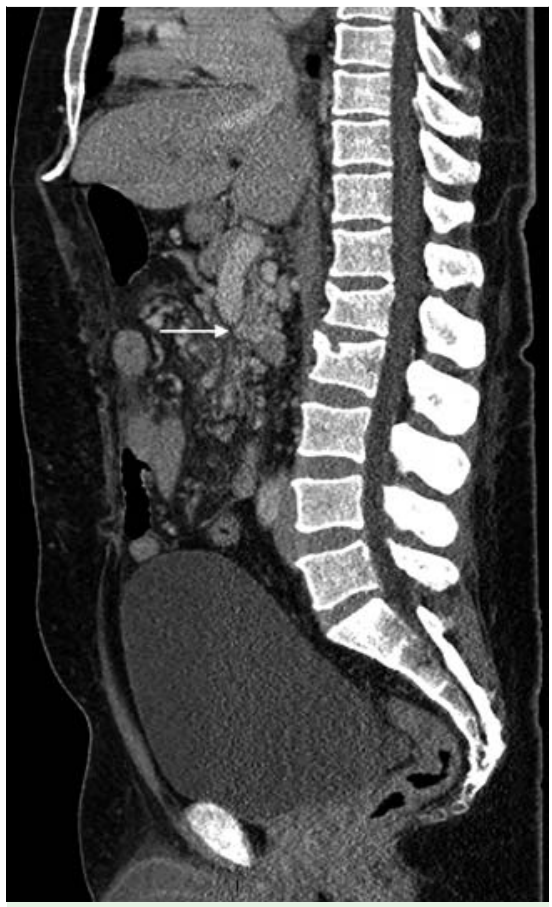

Fig. 3 Persistence of the superior mesenteric vein occlusion (arrow) 2 weeks after closure of the Petersen space.

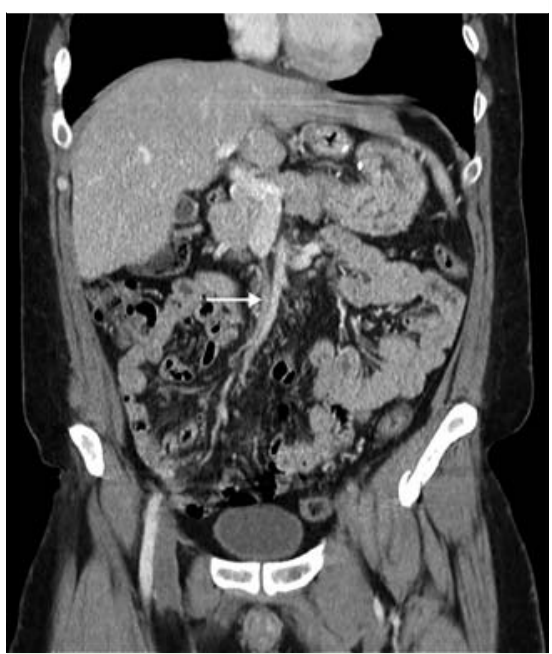

Fig.4 Repermeabilization of the superior mesenteric vein (arrow) after 3 months of anticoagulant treatment.

\section{Carmen Musala ${ }^{1}$, Jean Closset ${ }^{2}$, Patrizia Loi ${ }^{2}$, Emmanuel Coppens ${ }^{3}$, Jacques Devière ${ }^{1}$, Arnaud Lemmers ${ }^{1}$}

${ }^{1}$ Department of Gastroenterology, Hepatopancreatology and Digestive Oncology, Université Libre de Bruxelles, Erasme Hospital, Brussels, Belgium 2 Department of Abdominal Surgery, Université Libre de Bruxelles, Erasme Hospital, Brussels, Belgium

${ }^{3}$ Department of Radiology, Université Libre de Bruxelles, Erasme Hospital, Brussels, Belgium 


\section{References}

1 Perakh S, Soto E, Merola S et al. Diagnosis and management of internal hernias after laparoscopic gastric bypass. Obes Surg 2007; 17: $1498-1502$

2 Elms L, Moon RC, Varnadore $S$ et al. Causes of small bowel obstruction after Roux-en-Y gastric bypass: a review of 2,395 cases at a single institution. Surg Endosc 2014; 28 : $1624-1628$

3 Paroz A, Calmes JM, Giusti V et al. Internal hernia after laparoscopic Roux-en-Y gastric bypass for morbid obesity: a continuous challenge in bariatric surgery. Obes Surg 2006; 16: $1482-1487$
4 Brolin R, Kella V. Impact of complete mesenteric closure on small bowel obstruction and internal mesenteric hernia after laparoscopic Roux-en-Y gastric bypass. Surg Obes Relat Dis 2013; 11: 850-854

\section{Bibliography}

DOI http://dx.doi.org/

10.1055/s-0034-1391234

Endoscopy 2015; 47: E83-E84

(c) Georg Thieme Verlag KG

Stuttgart · New York

ISSN 0013-726X
Corresponding author

\section{Carmen Musala, MD}

Erasme Hospital

Université Libre de Bruxelles

Department of Gastroenterology,

Hepatopancreatology and Digestive Oncology

Route de Lennik, 808

Brussels 1070

Belgium

Fax: +32-2-5554802

Carmen.Musala@erasme.ulb.ac.be 